\title{
Zur Neuverhandlung konstituierender Grenzen: Textkritische Analyse und demokratietheoretische Interpretation der Erzählung vom Goldenen Kalb
}

\section{On the Renegotiation of Constitutive Borders: Textual Criticism and Interpretation of the Story of the Golden Calf through the lens of democratic theory}

\section{LAURIN MACKOWITZ, GraZ}

Zusammenfassung: Die politische Grenze, die in der Erzählung vom Goldenen Kalb schematisch gesetzt wird, ähnelt zeitgenössischen autoritären wie auch demokratischen Strategien, Macht durch gesellschaftliche Spaltungen auszuüben. In Bezug auf die Überlagerung politischer Differenzen mit moralischen und religiösen Werturteilen, die Verabsolutierung von Privilegien und die Verschleierung des Gesetzes scheint die in der Episode vom Goldenen Kalb erzählte Spaltung systematische Parallelen zu gegenwärtigen Praktiken innergesellschaftlicher Grenzziehung aufzuweisen. Die Aktualität der Berufung auf religiöse Erzählungen, rhetorische Polarisierungen oder antagonistische Symboliken macht die Kritik und Analyse der politisch-theologischen Sakralisierung politischer Differenzen zu einem wesentlichen Gegenstand der Aufklärung über die religiösen Grundlagen politischer Gewalt. Der Artikel interpretiert mit Verweis auf textkritische Analysen der Erzählung die Abspaltung der Leviten vom restlichen Volk als kultisch inszenierte militärische Aktion zur Herstellung und Befestigung von Privilegien durch Grausamkeit, den Tanz um das Goldene Kalb als Strategie der Konsolidierung einer durch die Abwesenheit von Gesetz und Führung verunsicherten Gemeinschaft und die Konstruktion gesellschaftlicher Antagonismen als Kompensation für die Verschleierung des Gesetzes. Abschließend wird diskutiert, inwiefern diese Strategien auch in Demokratien zentrale Merkmale der Konstituierung kollektiver Identitäten, Privilegien und Gewaltbeziehungen sind und ob es in Demokratien auch Raum für die Neuverhandlung innergesellschaftlicher Grenzen gibt. 
Schlagwörter: Goldenes Kalb, Exodus 32, Politische Theologie, Grenze, Demokratie

Abstract: The Story of the Golden Calf draws a political border in a way that resembles contemporary authoritarian (as well as democratic) strategies of exerting power by means of social division. The division narrated in the Story of the Golden Calf appears to show systematic parallels with current practices of demarcation, particularly regarding discourses that superimpose political differences with moral and religious value judgements and interpret privileges as absolute. In view of recent political appeals to religious narratives for reasons of rhetorical polarisation as well as considering political and juridical practices that conceal the law, the critique of the religious foundations and antagonistic symbolisms of political violence seems to be pertinent. This article interprets the separation of the Levites from the rest of the people as a military action that establishes privileges through cruelty. Textual criticism and the analysis of the sacralisation of political differences from the perspective of political theology indicates in which way, the dance around the golden calf is a consolidation strategy that confronts the social instability caused by the obfuscation of the law and the absence of leadership. Finally, the article discusses to what extent the construction of social antagonisms are central features of collective identities and whether democracy has room for the renegotiation of social divisions.

Keywords: Golden Calf, Exodus 32, Political Theology, Border, Democracy

„Bekanntlich währt ein Fest nicht lange, Beim ersten Geräusch an der Tür stieben die beiden Ratten auseinander. Es war nur ein Geräusch, und doch eine Botschaft - wie eine Nachricht, die Panik sät.“ (Michel Serres, Der Parasit, 1987, 11)

„Weißt Du auch, daß Zeiten vergehen werden und daß die Menschheit durch den Mund ihrer Weisheit und Wissenschaft verkünden wird, daß es kein Verbrechen gibt, folglich auch keine Sünde, sondern nur Hungernde?" (Dostojewski, Die Brüder Karamasow, 2016, 407)

\section{Einleitung}

Im Kontext einer politischen Theorie der Grenze kommt der Exodus-Erzählung besondere Bedeutung als Sammlung einer Reihe von Ein- und Ausschließungsmechanismen zu: Der Text motiviert die Befestigung von Grenzen, wenn er davon berichtet, wie Moses in die „wahre“ Familie hineingeboren (Ex 2,1-4), aber von der "falschen“ erzogen wird (Ex 2,5-10). Türpfosten werden mit Blut bemalt, um dem Tod aller Erstgeborenen zu 
entkommen (Ex 12,12-13). Gott markiert, indem er das Heer des Pharaos im Roten Meer ertränkt, einen Punkt ohne Wiederkehr (Ex 14,19-31). Jene Grenze, von deren Errichtung die Erzählung vom Goldenen Kalb (Ex 32) handelt, ist in einem besonderen Sinn politisch, da hier von der Spaltung einer Gemeinschaft und der Gründung einer Zweiklassengesellschaft berichtet wird, die sich nicht durch den Ausschluss einer amorphen Masse von Nichtmitgliedern, sondern durch eine innere Grenze konstituiert.

Die Exodus-Erzählung kommt am Fuß des Sinai zu einem narrativen Höhepunkt, als zwischen den vor der ägyptischen Unterdrückung geflohenen Israeliten eine Grenze gezogen wird, die das „Volk“ bzw. die Menge der aus Ägypten Entkommenen in zwei Gruppen spaltet, in die „immer schon Schuldigen“ und in ihre „Richter“. Womöglich besteht gerade in dieser Teilung der Unterschied zwischen einer Gemeinschaft der Verwandten und Freundeskreise - einer, wie Maurice Blanchot in Die uneingestehbare Gemeinschaft $(2007,57)$ schreibt, „brüderlich anonymen und unpersönlichen Bewegung“ - und einer politischen Gesellschaft voneinander getrennter Subjekte. Einer politischen Gesellschaft, die sich „über Praktiken der Unterwerfung“ konstituiert, wie Michel Foucault in Von der Freundschaft als Lebensweise argumentiert (1984, 138), oder in einen „Kampfplatz der Öffentlichkeit“ eintritt, wie Helmuth Plessner in Grenzen der Gemeinschaft: Eine Kritik des sozialen Radikalismus $(2003,82)$ schreibt.

Die von Moses und den Leviten gesetzte Grenze unterscheidet sich in ihrem spezifisch politischen Sinn von anderen in der jüdischen Tradition verankerten familiären, religiösen oder territorialen Begriffen der Grenze. In der Thora sind Grenzen, Brücken und Passagen häufige Motive. Schon der Name Iwri, Hebräer, erhält, wie Liliana Ruth Feierstein in From the Other Side of the River. Borders in Jewish Culture and History $(2011,108)$ schreibt, seine Bedeutung von denjenigen, die „von der anderen Seite des Flusses kommen“, Abraham überquert den Jordan nach Kanaan, Jakob kämpft mit Gott am Flussbett, Moses überquert das Rote Meer. Selbst wenn diese Flüsse auch geographische Grenzen markieren, das Trennende und das Zu-Überbrückende des Flusses wird im Jüdischen vor allem mit religiösen, ethischen und politischen Bedeutungen konnotiert. Beispielsweise befindet sich am Eingang jüdischer Häuser die mesusa, eine in den Türpfosten eingelassene Schriftkapsel, in der auf einem Pergament das jüdische Glaubensbekenntnis und das Wort schaddaj, Hüter der Pforten Israels, geschrieben stehen. Während des Sabbats, der durch die hawdala oder Trennung beendet wird, dürfen sich Juden nur im techum shabbat, in den Grenzen des Sab- 
bats, bewegen. Ha'Mavdil, „der, der trennt“, und bein kodesh le'chol, „der, der zwischen Heiligem und Profanem unterscheidet“, sind Namen Gottes. Cherem meint den Ausschluss aus der jüdischen Gemeinschaft (ebd.). Im Unterschied zu dieser Vielfalt religiöser, kultureller und sozialer Grenz-Begriffe wird in der Erzählung vom Goldenen Kalb eine in einem spezifischen Sinn politische Grenze, eine für die Gründung von Gesellschaft notwendige Trennung beschrieben.

Die Episode vom Goldenen Kalb ist in ihren Grundzügen schnell nacherzählt: Nachdem die Israeliten aus Ägypten entkommen sind, marschieren sie unter Entbehrungen durch die Wüste, um schließlich am Berg Sinai anzukommen. Gott verkündet die Zehn Gebote und das Bundesbuch. Die Israeliten erklären einstimmig zu gehorchen, Moses steigt auf den Berg, um die Steintafeln als Urkunde des Bundesschlusses zu empfangen. In seiner Abwesenheit fordern die Israeliten von Aaron ihnen neue Götter zu machen und Aaron gießt das Goldene Kalb. Gott wird darüber zornig und droht das Volk zu vernichten. Moses besänftigt ihn und steigt vom Berg herab. Im Lager sieht er die Israeliten um das Kalbbildnis tanzen und wird nun selbst zornig. Er zerbricht die Steintafeln und zerstört das Kalb. Anschließend spaltet Moses das Volk in zwei Teile, die Schuldigen werden zu Tausenden von den Strafenden hingerichtet. Den Überlebenden droht Gott ihre zukünftige Bestrafung an.

In dieser für die politische Interpretation der Exodus-Erzählung zentralen Episode vom Goldenen Kalb wird eine politische Strategie zur Errichtung und Stabilisierung innergesellschaftlicher Grenzen verdichtet und im Sinne einer Politischen Theologie politischer Grenzziehung sakralisiert. Der Begriff der Politischen Theologie (theologia civilis) meint hier - in Abgrenzung zur „dichterischen Rede von Göttlichem“ (theologia fabularis) und dem „auf das wahre Wesen des Göttlichen gerichtete Gott-Sagen der Philosophen“ (theologia naturalis) (Wacker und Manemann 2016, 15) - das Ineinandergreifen säkularer und sakraler Politik, die „Theologisierung der Politik und die Politisierung der Religion“ (Wiedenhofer 1976, 11), die Zurückführung einer politischen Theorie, Doktrin oder Position auf „die göttliche Offenbarung“ als „höchste Autorität und letzte Grundlage“ (Maier 2006, 13), wobei sie Aussagen „über den Status, die Legitimation, Aufgabe und evtl. Struktur der politischen Ordnung“ (Böckenförde 1985, 19) mit Bezug auf religiöse Narrative, Topoi oder Glaubensinhalte untermauert.

Im Kontext der in der Episode vom Goldenen Kalb begründeten gewaltsamen politischen Spaltung ist der in der Neuzeit aufkommende pole- 
mische Gebrauch des Begriffs der Politischen Theologie bedeutsam. Niccolò Machiavelli bemerkt, Religion würde „Heere in Gehorsam, das Volk in Eintracht halten“ $(1977,44)$, Baruch Spinoza schreibt, monarchische Regierungen würden aus Eigeninteresse die Menschen „unter dem schönen Namen Religion“ täuschen und mit Furcht „im Zaume“ halten, „damit sie für ihre Knechtschaft kämpfen, als sei es für ihr Heil, und damit sie es nicht für eine Schande, sondern für die höchste Ehre halten, für den Ruhm eines Menschen Blut und Leben hinzuopfern“ $(1984,6)$. Für Michail Bakunin bedeutet „politische Theologie“ nicht nur eine Technik zur Herstellung von Uniformität und Patriotismus, der Glaube an eine göttliche Autorität ist die eigentliche Ursache aller Unterdrückung $(1973,214)$. Die Aktualität der Berufung auf religiöse Glaubensinhalte, Erzählungen oder Vorschriften zur Rechtfertigung von Gewalt und Unterdrückung sowie das Argument, auch in Demokratien müsse ein ansonsten rein imaginäres Recht politisch theologisch symbolisiert und verkörpert werden (Lefort 1999, 93), machen die Kritik und Analyse des Fortwirkens Politischer Theologie zu einem wesentlichen Gegenstand der Aufklärung über die religiösen Grundlagen politischer Gewalt.

Im Gegensatz zu Carl Schmitts Bejahung der Politischen Theologie zur Herstellung politischer Kohärenz und Handlungsfähigkeit - „Kein politisches System kann mit bloßer Technik oder Machtbehauptung auch nur eine Generation überdauern. Zum Politischen gehört die Idee, weil es keine Politik gibt ohne Autorität und keine Autorität ohne ein Ethos der Überzeugung“ $(1925,23)$ - wird im Folgenden die Politische Theologie der Episode vom Goldenen Kalb hinsichtlich der Sakralisierung politischer Grenzen kritisiert. Diese Kritik betrifft auch die von der sogenannten „neuen politischen Theologie“ versuchte Verwandlung und Neuprägung des Begriffs (Metz 2016, 68). In diesem Sinn hinterfragt die politische Philosophie der Exodus-Erzählung die ihr eingeschriebenen politischen Aussagen und die durch oder nach ihrem Vorbild konstituierten politischen Autoritäten, Hierarchien, Identitäten und Strategien. Die philosophische Kritik Politischer Theologie dringt dabei, wie Walter Schweidler im Anschluss an René Girard argumentiert, zum „Kern politischen Denkens“ vor: zur Aufklärung der Verschleierung der am Anfang jeder politischen Ordnung stehenden „ursprünglichen tödlichen Gewalt“ durch deren „Sakralisierung“ (2011, 48f.).

Mit Blick auf gegenwärtige politische Strategien, Macht durch politische Grenzen, rhetorische Polarisierungen oder antagonistische Symboliken auszuüben, erscheint es daher wesentlich zu untersuchen, inwieweit zeitgenössische innergesellschaftliche Grenzziehungen durch religiöse Nar- 
rative untermauert oder sakralisiert werden. Der wachsende Einfluss ethno-nationalistischer Politik und deren Absichten, innere und äußere Feinde zu konstruieren und eine rigide Sicherheits- und Überwachungspolitik zu forcieren, wie sie u.a. in dem von Heinrich Geiselberger herausgegebenen Sammelband Die große Regression. Eine internationale Debatte über die geistige Situation der Zeit (2017) beschrieben wird, zeigt, dass gegenwärtige Identitätspolitiken an die Erzählung vom Goldenen Kalb und deren Muster der Ausschließung, Einschließung und Polarisierung anknüpfen.

So zielt die Festungsmetaphorik in der europäischen Außen- und Migrationspolitik nicht auf die Verhinderung von Mobilität, sondern auf die Konsolidierung einer brüchigen gesamteuropäischen Identität und Gouvernementalität. Diesbezüglich schreiben auch Dimitris Papadopoulos, Niamh Stephenson und Vassilis Tsianos in Escape Routes: Control and Subversion in the 21. Century, „borders [...] are erected wherever there is a need to solve and to organise social space and political governance“ (2008, 27). Analog dazu beabsichtigt auch die offen rassistische Rhetorik, mit der für den Bau der Grenzmauer zwischen Mexiko und den USA geworben wird, nicht allein Eindringlinge fernzuhalten. Vielmehr dient das Spektakel des Mauerbaus der Zementierung weißer, männlicher oder evangelikaler Privilegien und der Illusion, US-Präsident Donald Trump könne „souverän“ agieren, wenn es darum gehe, eine fiktive ökonomische und moralische Vorherrschaft der USA zu verteidigen (vgl. Ernst 2020, 242). Solche irreleitenden Evokationen politischer Macht schließen an Carl Schmitts Aktualisierung des von Jean Bodin zur Verteidigung absoluter monarchischer Gewaltausübung gebrauchten Begriffs der Souveränität an. Souverän sei dasjenige Subjekt, das „über den Ausnahmezustand“ $(1934,11)$ entscheide und damit „außerhalb der normal geltenden Rechtsordnung“ stehe (ebd., 13). Gegen eine solche Heroisierung singulärer Willensentscheidungen argumentiert Friedrich Balke, „Souveräne operieren immer schon, also zu allen Zeiten, in Netzwerken mit verteilter Handlungsmacht, die ihnen eine strukturell überlegene, aber letztlich doch niemals ,un-bedingte“ Gewalt verschaffen“ $(2009,15)$. Die autoritäre und nationalistische Rhetorik des Populismus verkennt die Interdependenz politischer Prozesse in größeren kulturellen und gesellschaftlichen Zusammenhängen. Ein solcher Isolationismus führt dabei zu einer verhärteten sozialpolitischen Gemengelage, die Torben Lütjen als Zerfall politischer Klassen in segmentierte „Echokammern“ beschreibt; in Folge sozialer Absonderung und Ausgrenzung würde der für Demokratien entscheidende produktive und an Sachfragen orientierte „Streit“ verhindert werden, indem der 
politische Gegner kriminalisiert und diskreditiert werde und „der anderen Seite die politische Legitimität insgesamt“ aberkannt würde (2016, 245).

Gegenwärtige politische Prozesse und Rhetoriken scheinen daher gerade in Bezug auf die Überlagerung politischer Differenzen mit moralischen und religiösen Werturteilen, die Verabsolutierung von Privilegien als Vorrechte des Souveräns, die Verschleierung und Sakralisierung des Gesetzes und die Erzwingung von Verpflichtung durch Spaltung beachtenswerte schematische Gemeinsamkeiten mit der Erzählung vom Goldenen Kalb aufzuweisen. Zwar scheint der Begriff des „biblischen Paradigmas“ politischer Grenzziehung für die fragmentarischen und womöglich auch schematischen Entsprechungen zwischen der biblischen Erzählung und modernen Grenzziehungsstrategien voreilig zu sein, die textkritische Analyse der Erzählung und deren Diskussion im Lichte zeitgenössischer Demokratietheorien wird aber deutlich machen, dass diese Erzählung eine idealtypische Vorgehensweise formuliert, nach der Gesellschaften dauerhaft und in sich selbst gespalten werden können: Als Erstes wird deren Identität als gefährdet inszeniert, zweitens wird mittels Gewalt eine Grenze zwischen zwei Gruppen gezogen und drittens werden Gesetze erlassen, die auf Dauer die Rechte und Pflichten der jeweiligen Gruppen definieren.

Im Folgenden werden Parallelen zwischen der in der Episode vom Goldenen Kalb erzählten Spaltung und gegenwärtigen Praktiken der Grenzziehung aufgezeigt und diskutiert, inwieweit auch demokratisch legitimierte Spaltungen dem biblischen Vorbild folgen: Durch das Ziehen innergesellschaftlicher Grenzen werden Gesetze durchgesetzt, politische Identitäten konstituiert sowie Privilegien und Diskriminierungen legitimiert. Zu diesem Zweck wird die Episode vom Goldenen Kalb zum einen aus textkritischer Sicht und zum anderen aus demokratietheoretischer Perspektive hinsichtlich der in der Exodus-Erzählung vorgeschlagenen Strategien zur Markierung, Exekution und Institution politischer Grenzen analysiert.

Als Erstes wird die Abspaltung der Leviten vom restlichen Volk als kultisch inszenierte militärische Aktion zur Herstellung und Befestigung von Privilegien durch Grausamkeit interpretiert. Als Zweites wird der Tanz um das Goldene Kalb als Strategie der Konsolidierung einer durch die Abwesenheit von Gesetz und Führung verunsicherten Gemeinschaft durch die Materialisierung eines abwesenden und begehrten gesellschaftlichen Zentrums beschrieben. Und als Drittes werden die Konstruktion eines gesellschaftlichen Antagonismus und die Reproduktion ethnischer und kultureller Grenzziehungen als Kompensation für die Verschleierung des Gesetzes diskutiert. 
Abschließend werden die Auswirkungen innergesellschaftlicher Grenzen für die Konstitution und Legitimierung kollektiver Identitäten, Privilegien und Gewaltbeziehungen beschrieben und dafür argumentiert, dass Strategien der Reproduktion und Legitimation innergesellschaftlicher Grenzen auch in Demokratien zentrale Merkmale der Konstituierung von Gesellschaft bleiben und materiell - wirtschaftlich wie physisch - durchgesetzt werden.

\section{Die Begründung von Privilegien durch Grausamkeit}

Die Episode vom Goldenen Kalb (Ex 32) nimmt eine zentrale Stellung innerhalb der Exodus-Erzählung ein und wird ihrem Höhepunkt, der Verkündung der Zehn Gebote, zugeordnet. Narrativ vorbereitet wird die Erzählung durch den Bericht über den Aufstieg Moses' und Joshuas zum Berg (Ex 24,12ff.), die detaillierte Beschreibung von Heiligtum und Ritus (Ex 25,1-31,17) und die Übergabe der Gesetzestafeln (Ex 31,18). Exodus 32 selbst beginnt mit der Schilderung des Zweifelns der Israeliten, ob Moses überhaupt vom Berg zurückkehren würde, und der Bitte an Aaron: „Komm, mach uns Götter, die vor uns herziehen“ (Ex 32,1). Aaron verlangt von allen die Herausgabe ihres goldenen Schmucks. Anschließend zeichnet und gießt er aus dem Gold das Kalb und es wird gefeiert (Ex 32,2-6). Jahwe bemerkt das Abfallen der Israeliten und will das gesamte Volk ausrotten, doch Moses bittet um Schonung (Ex 32,7-14). Moses und Joshua steigen vom Berg herab und vernehmen im Lager der Israeliten Lärm, Moses wird zornig und zerstört die von Gott geschriebenen Steintafeln (Ex 32,15-19). Dann zerstört er das Goldene Kalb und verhört Aaron (Ex 32,20-25). Schließlich tritt er an das Lagertor und ruft: „Wer für den Herrn ist, her zu mir!“ (Ex 32,26). Die Leviten versammeln sich um Moses, der ihnen befiehlt: „Jeder erschlage seinen Bruder, seinen Freund, seinen Nächsten“ (Ex 32,27). Die Leviten erschlagen viele aus der Menge und werden von Moses anschließend dafür gesegnet. Moses kehrt zum Berg zurück und verhandelt mit Gott, der verspricht, seine Abrechnung für den Verrat des Volkes auf unbestimmte Zeit aufzuschieben (Ex 32,34).

Neben Moses und Aaron können zwei Gruppen als Handlungsträger der Erzählung vom Goldenen Kalb unterschieden werden: „das Volk“ und „die Leviten“. Zwar wird der Gegensatz zwischen Moses und Aaron oft als ein zentrales Motiv der Erzählung identifiziert (vgl. u.a. Freud 1939; Schönberg 1954; Assmann 1998), für die Deutung der Erzählung hinsichtlich innergesellschaftlicher Grenzziehungsstrategien scheint es aber zielführender zu sein, stattdessen der Unterscheidung und Opposition zwischen Leviten und 
Volk nachzugehen. Den überbeanspruchten Gegensatz Moses - Aaron auBer Acht lassend, wird im Folgenden die Konstitution der beiden kollektiven Subjekte, der Leviten und der am Fuße des Berges versammelten Menge, die später zu einem mit der Schuld des Abfalls von Gott beladenen Volk gemacht wird, beschrieben.

Berühmte Kommentatoren der Erzählung haben die Leviten als Avantgarde identifiziert: Sigmund Freud vermutet in Der Mann Moses und die monotheistische Religion (1939, 488), dass die Leviten eine „einflußreiche, den anderen kulturell überlegene Minorität“ bilden, die, da es nur bei den Leviten „später noch ägyptische Namen“ gegeben habe, sich aus dem Gefolge Moses' zusammengesetzt hätten, das der hohe ägyptische Beamte mit sich gebracht habe, als er sich zu den Semiten begeben habe. Die zentrale Bedeutung der Erzählung vom Goldenen Kalb innerhalb des Exodus gibt auch Michael Walzer in Exodus und Revolution $(1995,100)$ Anlass dazu, die politische Philosophie des Exodus als Herrschaft einer Avantgarde über die Masse zu konzipieren. Solange das Volk nicht fähig sei, ohne Ermahnung und Strafe die Gesetze einzuhalten, müsse es einer Elite überlassen werden, an Stelle des Volkes zu entscheiden, schreibt Walzer. Zwei Ämter müssten darauf achten, dass die Gesetze und die Pflichten auch tatsächlich eingehalten würden, die Priester, die „für das Volk handeln“, und die Propheten, die „das Volk auffordern zu handeln“ (ebd.). Kritisch gegenüber diesen politisch-theologischen Institutionen gibt Baruch Spinoza in seinem Theologisch-politischen Traktat (1984, 271f.) zu verstehen, dass die Leviten vermutlich keine harmlosen Theologen, sondern beim Volk verhasst waren, da sie sich durch nichts anderes unterschieden als durch ihre Aufgabe, die Menge stellvertretend für den Zorn Gottes zu bestrafen.

Die textkritische Analyse des evangelischen Theologen Rainer Albertz $(2015,269)$ zeigt, dass die Strafaktion als militärische Aktion betrachtet werden muss, da die „Verwendung des Ausdrucks nāpal min in Exodus 32,28, [...] das ,Fallen (einer Anzahl) von“ Menschen im Krieg bezeichnet“. Diese Hinweise auf die blanke Gewalt, mit der die levitische Grenzziehung durchgesetzt wird, geben zu verstehen, dass einige sich der Bestrafung für ihr Abfallen vom Glauben entziehen können, während diejenigen, die Widerstand leisten, ohne weitere Diskussion getötet werden. Die Leviten sind Soldaten, die für ihre Henkersdienste durch Privilegien belohnt werden: „Füllt eure Hände mit Gaben für den Herrn!“ (Ex 32,29). Den anderen Überlebenden des Massakers gibt Moses die Asche des verbrannten Goldenen Kalbs zu trinken (Ex 32,20) und verurteilt und verflucht sie auf diese Weise, wie Al- 
bertz $(2015,277)$ schreibt. An dieser für den biblischen Zusammenhang von Sünde und Strafe zentralen Stelle wird die Metapher des Flusses benützt - so heißt es an anderer Stelle, Moses streute die Asche „in den Bach, der vom Berg herunterfließt“ (Dtn 9,21) -, um die Grenze zwischen der Menge vor der Sünde und dem von nun an auf ewig verunreinigten und verfluchten Volk narrativ zu unterstreichen.

Josef Schmidt beschreibt die Leviten in Jakob - Echnaton - Moses $(2013,317)$ als „Gefolgsleute Joshuas“, die für ihren Einsatz gesegnet und als Priester eingesetzt werden. Als Moses das Volk mit dem Aufruf „Wer für den Herrn ist, her zu mir!“ in zwei Gruppen spaltet, scheiden sich die Freiwilligen und Eifrigen vom restlichen Volk, „da sammelten sich alle Leviten um ihn“ (Ex 32,26). Die Leviten sind, wie der Text zu verstehen gibt, diejenigen, die Moses’ Befehl gehorchen: „Zieht durch das Lager von Tor zu Tor! Jeder erschlage seinen Bruder, seinen Freund, seinen Nächsten“ (Ex 32,27). „Die Leviten taten, was Mose gesagt hatte. Vom Volk fielen an jenem Tag dreitausend Mann“ (Ex 32,28). Der Text bleibt im Gegensatz dazu vage in Bezug auf die Frage, wer ermordet werden solle, und lässt offen, wer mit den Getöteten gemeint sei. Dem Terror der Leviten erscheinen dadurch alle als gleich verdächtig, jeden kann jetzt oder auch später die Strafe treffen. Die Aktion der Leviten verdoppelt die Strafe, viele werden sofort hingerichtet, den anderen wird eine Bestrafung in der Zukunft angekündigt, womit einerseits jedes in Zukunft erfahrene Unheil als Strafe gedeutet werden kann und andererseits zur strengen Einübung der Gesetze ermahnt wird.

Die Strafaktion der Leviten und die anschließende Segnung und Verfluchung durch Moses stehen nicht nur außerhalb des zuvor beglaubigten Gebotes, „du sollst nicht morden“ (Ex 20,13), sie sind Ausdruck der jeder politischen Autorität und Hierarchie zugrundeliegenden Gewalt; der „Systeme von Grausamkeiten“, die, wie Friedrich Nietzsche in der Genealogie der Moral schreibt, Erziehung und Erinnerung nach dem Hauptsatz gestalten, „nur was nicht aufhört, wehzutun, bleibt im Gedächtnis“ (1999, 295). Die rituelle Bestrafung und Verfluchung des Volkes begründet die Bewahrung politischer Ordnung durch Furcht vor „Grausamkeit“, einer, wie Marcel Hénaff schreibt, „Intensivierung der Gewalt [...], dem Willen den Gegner durch physischen Schmerz leiden zu lassen und ihn über den Sieg hinaus durch Erniedrigung in Verzweiflung zu stürzen“ (2015, 12-13). Die einmalig exekutierte und kultisch inszenierte Grausamkeit verschafft der nun nur mehr angedrohten Gewalt Glaubwürdigkeit. 


\section{Die Bedürftigkeit des Volkes, das Kultbild und der Tanz}

Die Leviten begründen durch die rituelle und grausame Gewalt, mit der sie sich vom Volk abspalten, ihre sakrale, ökonomische, militärische und politische Vorrangstellung gegenüber einer zweiten Gruppe, die erst durch die Spaltung eine kollektive Identität erhält und als Gruppe der Schuldigen und zu Bestrafenden erscheint. Obwohl das Volk des Bundes, das sich in Exodus 24 zur Einhaltung der Gesetze verpflichtet und sich Gott unterwirft, „alles, was der Herr gesagt hat, wollen wir tun; wir wollen gehorchen“ (Ex 24,7), schon eine politische Identität formiert, nennt die spätere jüdische Tradition die Menschen, die sich am Fuß des Sinai versammelt haben und auf die Rückkehr Moses' warten, wie Gunther Plaut in seiner progressiven Jüdischen Auslegung der Tora (2000, 329) festhält, eine „Menge allerlei Leute“.

Die sich gleich „versündigende“ Menge ist nicht oder noch nicht das auserwählte Volk, sie ist die nicht weiter definierte Menge der aus Ägypten und vor ihrer Unterdrückung geflohenen Menschen. Befreit von der Unterdrückung als Sklaven in Ägypten und nach einem langen, schweren Weg durch die Wüste wartet diese Menge am Fuß des Sinai darauf, dass ihr Führer und sein Gott zurückkehren. Nachdem Moses vierzig Tage nicht zurückgekehrt ist, werden die Menschen ungeduldig und fragen nicht nur danach, was getan werden müsse, sie wollen wissen, wen sie anbeten sollen: „Komm, mach uns Götter, die vor uns herziehen“ (Ex 32,1). Die Menschen am Fuß des Sinai werden als unsicher und ungeduldig stilisiert, um das Handeln ihrer Führer, sowohl Aarons Verführung als auch Moses' Bestrafung, zu rechtfertigen. Aaron weicht Moses' Zorn mit den Worten aus: „Du weißt doch, wie böse das Volk ist“ (Ex 32,22), und Moses stimmt zu: „Denn Aaron hatte es verwildern lassen, zur Schadenfreude seiner Widersacher“ (Ex 32,25).

Auch hier entwickelt der biblische Text ein Argumentationsschema zur Rechtfertigung autoritärer Politik: Ungeordnete Menschenmengen würden von einer Disposition zu Ungeduld und Unsicherheit bestimmt, weshalb die Abwesenheit starker Führungspersönlichkeiten Devianzen von einer als gerecht und göttlich inszenierten Ordnung und Regressionen in eine als überwunden stilisierte ungerechte Gesellschaft verursache und folglich eine als tugendhaft identifizierte Elite über die wankelmütige Menge herrschen müsse (vgl. La Boétie 1924, 9; Spinoza 1984, 6). Das in der Episode vom Goldenen Kalb prototypisch formulierte Argument ähnelt dabei dem Diskurs des Großinquisitors in Fjodor Dostojewskis Die Brüder Karamasow (2016, 409): Die Menschen würden niemals Freiheit und Brot gleichzeitig haben, da sie, so kleinmütig und lasterhaft, wie sie seien, nicht miteinander teilen 
könnten, weshalb die Führer der Kirche sie bevormunden und regieren, ihnen vor allem aber ein Objekt für ihr individuelles und kollektives Bedürfnis, jemanden anzubeten, geben müssten.

Dieses Bedürfnis erfüllt das materielle Bild des Goldenen Kalbs in Konkurrenz mit dem unsichtbaren Gott Moses, indem es nicht etwa eindeutig als Kultbild (pesel), Gestalt (tșmûnah), Gottesbild ('așabîm) benannt wird, wie Schmidt $(2013,317)$ referiert, sondern unscharf und diffus mit dem, nunmehr zur Metapher geronnenen, Namen „Goldenes Kalb“ (egel); einem Namen, der über Religion und Politik hinaus in vielen Kontexten Bedeutungen transportiert und Gefühle evoziert. Der Metaphern-Körper des Goldenen Kalbs fungiert dabei wie ein Gefäß oder Platzhalter, der alles Obszöne, Sündhafte und Abweichende in sich aufnimmt. Auch Horst Seebass betont in Mose und Aaron, Sinai und Gottesberg (1962, 34), das Goldene Kalb gewinne diese Bedeutung, indem es narrativ zu den Tafeln des Bundesschlusses in Opposition gestellt werde. Aaron zeichnet das Goldene Kalb lediglich „mit einem Griffel“ (Ex 32,4), die Tafeln des Bundesschlusses sind aber mit „Gottes Schrift“ (Ex 32,16) geschrieben.

Dabei unterscheiden sich die jeweiligen Kultgegenstände, Kalb und Gesetzestafeln, gar nicht so sehr. Beides sind abstrakte Zeichen einer Gottesverehrung, die auch materiell repräsentiert werden. Die zwei Tafeln der Bundesurkunde sind wie das Goldene Kalb ein Kultobjekt, das im Allerheiligsten der Bundeslade aufbewahrt wird. So beanstandet Aaron, dass nicht nur das Kalb, sondern auch die Tafeln „eine körperliche Befleckung der vollkommenen Reinheit sind“, wie Plaut schreibt (2000, 335). Der als Gegensatz zwischen Moses und Aaron konstruierte Konflikt zwischen der Hingabe an einen allmächtigen, unsichtbaren Gott auf der einen Seite und der Notwendigkeit eines sichtbaren Kultbildes auf der anderen bzw. der „Kluft zwischen einem Ideal, das das Volk verehren kann, und der reinen Vorstellung des Göttlichen" (ebd.), sollte daher nicht überbewertet werden. Auch Schmidt $(2013,316)$ argumentiert, dass die Anbetung des Kalbs keinen Rückfall in den Viel-Götter-Glauben darstelle. Die Israeliten würden keinem anderen Gott huldigen und das Kalb mit dem Namen Jahwe anbeten, wofür der semantisch als numerischer Singular zu identifizierende Plural Elohim stehe, weshalb die in Exodus 32,8 formulierte Kritik sich nicht auf ein Abfallen von Gott, sondern auf „die Verdinglichung Gottes“ beziehe (ebd.).

Folglich geht es der auf Moses' Rückkehr wartenden Menge am Sinai nicht um einen Führer oder einen Gott, sondern um ein Bild, um eine körperliche Manifestation ihres Glaubens und ihrer gesellschaftlichen Einheit. 
Die Menschen wollen einen Götzen, sie haben Sehnsucht nach einem Massenideal, das, wie Elias Canetti in Masse und Macht $(1978,12)$ vermutet, eher dem Bedürfnis des vereinzelten „berührungsfürchtigen“ Individuums, sich in die Masse zu befreien, entspricht als den Schuldgefühlen gegenüber einer göttlichen oder mosaischen Autorität. Das Goldene Kalb soll den Mittelpunkt der Gemeinschaft besetzen und dem panischen Zerfall zentripetaler Vereinzelung mithilfe des Tanzes um ein gesellschaftliches Zentrum entgegenwirken (ebd., 25).

Deleuze und Guattari haben in Tausend Plateaus. Kapitalismus und Schizophrenie II (1992, 424) einen solchen Gemeinschaft konstituierenden Tanz als dreistufiges Ritornell analysiert und dessen erste Stufe wie folgt beschrieben: „Ein Kind, das im Dunklen Angst bekommt, beruhigt sich, indem es singt. Im Einklang mit seinem Lied geht es weiter oder bleibt stehen. Hat es sich verlaufen, versteckt es sich, so gut es geht, hinter dem Lied, oder versucht, sich recht und schlecht an seinem Lied zu orientieren. Der Rhythmus und die Melodie dieses Lieds sind so etwas wie der erste Ansatz für ein stabiles und beruhigendes Zentrum mitten im Chaos.“ Das Ritornell ist auf dieser Stufe der „gefestigten Haltung“ temporär, labil und mobil (ebd.). In seiner zweiten evolutionären Stufe zieht es einen Kreis durch eine „Klangmauer“, die ein „Zuhause“ umgibt, ein Territorium markiert und die „Aktivität[en] des Selektierens, Eliminierens und Extrahierens“ instituiert (ebd.). Die dritte Stufe des Ritornells „öffnet den Kreis“, um in „umherirrenden“, aber letztlich „zentrifugalen“ Fluchtbewegungen zu improvisieren und das Außen hereinzulassen (ebd., 425).

Das „Fest zur Ehre des Herrn“ (Ex 32,5), mit dem das Goldene Kalb eingeweiht werden soll, kann anhand von Deleuze und Guattaris schematischer Reflexion als erste Stufe des Ritornells verstanden werden. Die Worte, mit denen das Goldene Kalb begrüßt wird: „Dies ist dein Gott, Israel, der dich aus dem Land Ägypten herausgeführt hat“ (Ex 32,4), bezeichnet Albertz (2015, 270f.) als „Kultruf“ und „Identifikationsformel“, die wie das Lied die „Kultgemeinde bei der Prozession“ vereinigen. Dabei stellt Albertz außerdem klar, dass aus textkritischer Perspektive die Erzählung vom Tanz um das Kalb keine orgiastischen oder sexuellen Konnotationen weckt, sondern sprachlich einem kindlichen Kontext entlehnt sein müsse, da das entscheidende Verb in: „dann standen sie auf, um sich zu vergnügen“ (Ex 32,6), sahaq, als Lachen übersetzt werde und „das Spielen der Kinder (Gen 2,9; Sach 8,5) bzw. das Singen (Jer 15,17) und Tanzen (Ri 16,25; Jer 31,4) der Erwachsenen“ meine. Der Tanz um das Goldene Kalb stabilisiert Sozialität 
durch körperliche, spielerische und kommunikative Praktiken der Selbstversicherung einer verunsicherten Gemeinschaft.

\section{Das abwesende Gesetz und die anwesende Spaltung}

Die Strafaktion der Leviten erscheint in dem von Deleuze und Guattari konzipierten Ritornell-Modell als Aus- und Einschluss der zweiten Stufe: „Die Kräfte des Chaos werden, soweit als möglich, draußen gehalten “ $(1992,424)$. Auch Canetti betont den Aspekt der Selbstversicherung, wenn er argumentiert, es sei die „sicherste und oft die einzige Möglichkeit für die Masse, sich zu erhalten“, eine „zweite[] Masse, auf die sie sich bezieht“ (1978, 67), zu konstruieren. Die durch das Fehlen eines sichtbaren gesellschaftlichen Zentrums, durch den Rückzug Moses auf den Sinai und das Verbrennen des Goldenen Kalbs, verunsicherte Gesellschaft wird durch die Spaltung konsolidiert; die anwesende Grenze kompensiert das abwesende Zentrum. Gewalt besetzt die leere Stelle des Gesetzes.

In diesem Sinn vergleichbar gründet auch die Imagination und Instituierung nationaler Identitäten nicht auf einem anwesenden gesellschaftlichen Zentrum, sondern auf konstruierten, markierten und exekutierten, Differenzen. So bezeichnet Étienne Balibar in Der Schauplatz des Anderen: Formen der Gewalt und Grenzen der Zivilität $(2006,144)$ die Teilung in eine „empirische[] Nation“ und eine „transzendentale Nation“ als das Fundament der „Republik“. Diese „fiktive Ethnizität“ der transzendentalen Nation werde „von den hegemonialen Institutionen hervorgebracht und reproduziert", so Balibar (ebd.). Sie sei „die Krönung des gesamten Normierungs- und Ausgrenzungssystems“ und „das stärkste historische Instrument [...], um den Raum der Freiheiten durch Formalisierung der sozialen Kämpfe und demokratischen Forderungen zu durchstaatlichen" (ebd., 312). Staatliche Macht bestehe folglich in der effektiven Steuerung von Ausgrenzungsprozessen durch die erziehende Reproduktion ethnischer und kultureller Grenzziehungen (ebd.).

Die Autoren der Exodus-Erzählung konstruieren eine transzendente Nation, wie sie Balibar denkt, durch Spaltung, um zu garantieren, dass deren Mitglieder auch im Exil und unter Fremden nicht von den von ihnen definierten Glaubensüberzeugungen, Gesetzen und Grenzen abfallen. Die Spaltung der Menge in Leviten und Laien soll gegen die Kontingenz historischer sozialer und politischer Ordnungen und in Ergänzung zur unsichtbaren und vorläufigen Instanz des Gesetzes Gesellschaft durch die Errichtung einer in- 
neren Grenze konstituieren und instituieren. Durch die Strafaktion der Leviten kann das Gesetz als transzendenter Grund unsichtbar bleiben, während die Spaltung der Gesellschaft durch Sünde und Strafe in Erscheinung tritt. Es reicht, die Grenze zu exekutieren, um so Differenzen zu schaffen und diese im Folgenden (d.h. bis in die heutige Zeit) durch die formelhafte, und quasi als Präzedenzfall dienende, Erzählung der Episode vom Goldenen Kalb zu legitimieren.

In Bezug auf solche essentialistischen Identitätskonstruktionen zeigt die Interpretation der Episode vom Goldenen Kalb, auf welche Art und Weise politische Grenzen innerhalb von Gesellschaften instituiert und in einem zweiten Schritt durch deren Verschleierung und Verdoppelung einer kritischen Befragung entzogen werden. Eine Unstimmigkeit innerhalb der narrativen Abfolge von Befreiung, Bundesschluss und Stiftung des Gesetzes gibt zu erkennen, dass die Erzählung vom Goldenen Kalb nicht nur eine gesellschaftliche Spaltung, sondern auch eine Verdoppelung des Gesetzes begründen soll. Warum wird, nachdem die Israeliten bereits in Exodus 20 auf den Dekalog und die Gesetze eingeschworen werden, ein zweites Gesetz erlassen? Das erste Gesetz enthält den Dekalog und eine Reihe von Gesetzen des profanen Lebens, Moses schreibt es nach den Worten Gottes und teilt es den Israeliten mündlich mit (Ex 20,1-23,33). Das zweite Gesetz schreibt Gott mit seinem eigenen Finger auf die zwei Tafeln der Bundesurkunde (Ex 32,15 und 16). Gegenüber dem ersten Gesetz verpflichten sich die Israeliten freiwillig schon in Exodus 24,3; dem Bundesschluss, dem zweiten Gesetz, das die Kultvorschriften enthält, stimmen sie nicht zu, es wird erlassen.

In der Erzählung wird dieses Gesetz dem Zugang des Volkes entzogen, indem Moses die Gesetzestafeln zertrümmert und deren zweite Niederschrift für niemanden zugänglich in der Bundeslade aufbewahren lässt. Moses steigert, wie mit Michel Serres $(1987,62)$ argumentiert werden kann, durch diesen „Diebstahl an Information“ die Macht des zweiten Gesetzes, das selbst nicht in Erscheinung tritt. „Es genügt“, wie Serres in Der Parasit (ebd., 62f.) schreibt, „Gesetze zu erlassen und deren Kenntnis der Mehrzahl vorzuenthalten. Und das so sehr, daß die Macht letztlich nichts anderes ist.“ Das Gesetz wird zerstört, neu geschrieben, vor dem Volk versteckt und der Prüfung vorenthalten. Es wird dadurch transzendent, es wird der Macht der Menschen entrückt, und hängt in weiterer Folge wie ein Damoklesschwert über allen potentiell immer schon Schuldigen.

Claude Lefort hat die Verdoppelung des Gesetzes in ein abwesendes Gesetz und eine anwesende Teilung in Die Frage der Demokratie (1990, 
284) als „Konstitution eines gesellschaftlichen Raumes“ durch eine „doppelte[] Bewegung des Erscheinens und Verbergens“ erklärt: „Ein Erscheinen in dem Sinne, daß der Prozeß, durch den sich die Gesellschaft ordnet und durch ihre Teilungen hindurch vereinigt, sichtbar wird, Verbergung aber in dem Sinne, daß das generische Prinzip der Konfiguration der Gesamtgesellschaft verschleiert wird“ (ebd.). Politik erscheine nur durch ihre Teilungen, das Gesetz, das diese Teilungen fordere, bleibe aber abwesend und nicht verhandelbar. Die „Einheit der Gesellschaft“ könne, so Lefort, nur in der „Spaltung zwischen dem gesellschaftlichen Innen und Außen“ (ebd., 293) erfasst werden. In dem „Moment, in dem Gesellschaft sich ihrer Selbstidentität versichere“ (ebd.), also immer dann, wenn die Gesellschaft als ganze aufgefordert werde, ihre Macht zu zeigen, wird, Lefort zufolge, eine Grenze errichtet und ein Außen generiert.

Lefort führt diesen Antagonismus im Zentrum des Politischen auf den Erfinder des modernen politischen Denkens, Niccolò Machiavelli, zurück sowie auf dessen Unterscheidung zwischen den Noblen, die herrschen wollen, und dem Volk, das sich nicht beherrschen lassen will und sich gegen Unterdrückung wehrt. In der Republik führt der Antagonismus zum Agonismus, in der Demokratie, so Lefort, zur Institutionalisierung (ebd.). Ob eine Gesellschaft aristokratisch, demokratisch oder totalitär ist, entscheidet sich an der Frage, welche Antagonisten konstruiert und auf welche Art und Weise deren Widersprüche verhandelt werden. Der Antagonismus zwischen den Leviten und dem Volk unterscheidet sich gegenüber dem Agonismus der Renaissance jedoch hinsichtlich seiner kollektiven Dimension. Während die Spaltung der aus Ägypten befreiten Menge die bis heute bestehende religiöse Identität der Juden begründet, mündet der Wettkampf der florentinischen Fürstenfamilien, wie derjenige Castruccio Castracanis und Cesare Borgias, in die Agonie Einzelner, welche in Dante Alighieris (2001, 127ff.) Versen über den Todeskampf Ugolinos (Inferno XXXIII) ihren abschreckendsten Ausdruck gefunden hat: „Doch weint' ich nicht, und diesen Tag lang sagte / Ich nichts und nichts die Nacht, bis abermal / Des Morgens Licht der Welt im Osten tagte. / Als in mein jammervoll Verlies sein Strahl / Ein wenig fiel, da schien es mir, ich fände / Auf vier Gesichtern mein's und meine Qual.“ Der biblische Antagonismus betrifft im Gegensatz dazu ein Kollektiv, dessen Identität durch eine generationenübergreifende Verschuldung konstituiert wird. 


\section{Für Demokratie notwendige Grenzen demokratisch verhandeln und gestalten}

Mit Ausschlüssen wird auf Verunsicherung reagiert, die Grenzen, die dabei gezogen werden, konsolidieren Gemeinschaft aber immer nur vorläufig. Hannah Arendt betont in Vita Activa oder vom tätigen Leben $(2002,183)$, dass, obwohl „Schranken und Grenzen den niemals verläßlichen Rahmen her[stellen], in dem Menschen sich bewegen, ohne den ein Zusammenleben überhaupt nicht möglich wäre“, dieser Rahmen jedoch „oft noch nicht einmal stabil genug ist, um dem Ansturm zu widerstehen, mit dem jede neue Generation der Geborenen sich in ihn einschaltet". Grenzen werden andauernd gezogen, um Ordnungen herzustellen oder umzuwerfen. Dabei macht es allerdings einen großen Unterschied, welche Grenzen gezogen werden und mittels welcher Argumente diese legitimiert werden (ebd., 190).

Auch Chantal Mouffe argumentiert, dass ohne Grenzen und Ausschließungen kein politischer Bereich bestehen könne: In Agonistik. Die Welt politisch denken (2014, 24f.) greift sie Henry Statens Begriff des „konstitutiven Außen“ auf, um ihre These zu verdeutlichen: „Das Politische hat [...] von Anfang an mit kollektiven Formen der Identifikation zu tun, geht es in diesem Bereich doch stets um die Ausbildung eines ,Wir im Gegensatz zu einem ,Sie“." Identität sei nur möglich, „wenn sie als Differenz konstruiert“ (ebd.) werde. Gerade eine „pluralistische, liberale, demokratische Politik“ (ebd., 28) dürfe diese Grenzen nicht verleugnen, sondern müsse sie akzeptieren und den „Widerstreit demokratischer politischer Positionen“ fordern, um Konflikte zwischen „nicht verhandelbaren moralischen Werten oder essentialistischen Formen der Identifikation" (ebd., 29) zu verhindern.

Die Institutionen der Demokratie inszenieren solche unterschwellig fortwirkenden Antagonismen nicht, sie werden archiviert, weshalb sie in Form bürokratischer Ein- und Ausschließungsverfahren wiederkehren. Die Gewalt dieser Konflikte könnte, wie Oliver Marchart in Die politische Differenz (2010, 332f.) argumentiert, nur dann gezähmt werden, wenn „die grundlegende Antinomie der Demokratie" akzeptiert werde, der Widerspruch zwischen ihrem Durchsetzungsanspruch und ihrer Kontingenzakzeptanz - welche auch Antidemokratisches, Autoritäres und Anarchistisches einschließe. DemokratInnen müssten in diesem Sinne einer „Ethik der Selbstentfremdung " und „der Anerkennung [...] der nicht-selbstidentischen Natur jeder sozialen Identität“ folgen (ebd., 360). Eine solche Ethik plädiert für das Aushalten des Zerfalls nationaler oder religiöser Identitäten, nimmt dem Begriff der Solidarität seine paternalistische Konnotation assimilie- 
render Integrationsversuche eines gesellschaftlichen Außen und entschärft Konflikte, die wegen realer oder fiktiver Bedrohungsszenarien demokratische Gesellschaften zu spalten drohen.

Zwar argumentiert Jürgen Manemann in Exoduspolitik - Politik ex memoria passionis, Demokratien würden sich nicht durch Gewalt stabilisieren, „sondern durch die mannigfaltigen Formen des Sich-aneinander-Bindens, durch die Versprechen, Assoziationen und Verfassungen“ (2014, 340). Die nachhaltige Konstitution einer kollektiven politischen Identität durch Verschuldung und Bestrafung, wie sie in der biblischen Episode vom Goldenen Kalb erzählt wird, lässt aber vermuten, dass innergesellschaftliche Grenzen auch in Demokratien nicht restlos durch einen öffentlichen Diskurs kritisiert, überprüft und neu verhandelt werden können. Bezüglich der Frage, wie mit der Vielzahl innergesellschaftlicher Grenzen und Polarisierungen in zeitgenössischen Gesellschaften demokratisch umgegangen werden kann, legt eine kritische Sicht auf die levitische Strafaktion, die Verschleierung und die Verdoppelung des Gesetzes nahe, dass bestehende innergesellschaftliche Grenzen erst sichtbar gemacht werden müssen, bevor sie verhandelt werden können. Die „entscheidende Frage [...], um die es in demokratischer Politik geht“, lautet daher, wie auch Mouffe argumentiert, „nicht, wie man im Widerstreit der Interessen einen Kompromiss aushandelt oder wie man einen ,rationalen', das heißt alle einschließenden Konsens ohne jede Exklusion erzielt“ (2014, 31), sondern wie diesen Differenzen und Gegensätzen „Gestalt gegeben wird“ (ebd.).

Angesichts der durch transzendente oder sakralisierte Identitäten ausgelösten Grausamkeiten bleibt es aber fraglich, ob nicht jede neue Gestalt auch neue Gewalt impliziert und deshalb allenfalls negativ bestimmt werden sollte. Alain Badiou schreibt bezüglich der demokratischen Gestaltung des Fremden und Eigenen in Die Gegenwärtige Welt und das Begehren der Philosophie (2010, 30), dass „die starken Kategorien, die es erlaubten, das kollektive Subjekt zu begreifen, [...] ob es sich nun um Figuren der fortschreitenden Menschheit oder der großen Klassensubjekte handelt“, heute leer und unfähig seien, politische Ereignisse auszulösen, weshalb nur mehr das „Unmenschliche“ zum Ausgangspunkt nicht einer politischen, sondern einer ethischen Entscheidung - „im eigenen Namen vor dem Unmenschlichen“ (ebd.) - gemacht werden könne.

Auch Serres plädiert in einem unter dem Titel Aufklärungen (2008, 250) erschienenen Gespräch mit Bruno Latour dafür, neue, den Dekalog ergänzende Grenzen zu benennen: Das Gebot „Du sollst nicht töten“ müsse 
durch das Gebot „Du sollst dich nicht der Gewalt hingeben“ ergänzt werden. „I. Du sollst dich nicht der Gewalt hingeben, weder gegenüber diesem oder jenem, fremden oder nächsten Individuum noch gegenüber der globalen menschlichen Gattung. II. Du sollst dich nicht der Gewalt hingeben, weder gegenüber dem, was in deiner Umgebung kriecht und lebt, noch gegenüber dem Erdplaneten als ganzem. III. Und schließlich sollst du dich keiner Gewalt im Geiste hingeben; denn der Geist übersteigt, seit er in die Wissenschaft eingetreten ist, das Bewusstsein oder die Absicht und wird zum prinzipiellen Multiplikator der Gewalt" (ebd.).

Bezüglich der Gestalt dieser nur schwer fassbaren Grenzen, der Grenze vor dem „Unmenschlichen“ und der Grenze vor der „Hingabe“, oder besser der Duldung von Gewalt, legen Jacques Derridas in Die Schrift und die Differenz $(1972,351)$ nachzulesende Betrachtungen über Antonin Artauds „Theater der Grausamkeit“ nahe, dass selbst die ausgrenzungswürdigsten Abscheulichkeiten „bejaht“ werden müssen, d.h., dass sie „in ihrer vollen und notwendigen Unerbitterlichkeit“ und ,in ihrem verborgenen, zumeist vergrabenen, von sich selbst abgelenkten Sinn" gedacht und diskutiert werden müssen. Die Erzählung vom Goldenen Kalb zeigt dahingehend, dass gerade eine Grenzen setzende Gewalt dazu neigt, grausame kollektive Strafen zu verhängen und deren anschließende Begründung hinter religiösen oder moralischen Werturteilen zu verbergen. Um diese Gewalt sichtbar zu machen und damit die Frage, wie in Demokratien Grenzen gesetzt werden sollen, verhandelbar zu machen, bedarf es nicht nur einer eindeutigen Verurteilung von willkürlicher Polizeigewalt und politisch motivierter Desinformation. Die Schuld und Sünde, die Agonie, die Unmenschlichkeit, das Grauen und das Töten, die durch politische Grenzen erzeugt werden, müssen, wie Derrida nahelegt (ebd.), regelmäßig durch die Tragödie und Komödie verhandelt und entschärft werden, da diese nur schwer fassbaren Negativitäten ansonsten von leichter vorstellbaren Antagonismen besetzt werden. Die Erzählung vom Goldenen Kalb, als Tragödie gelesen, weist darauf hin, dass Grenzen, die aus Verunsicherung gesetzt werden, Ausschließungen erzeugen, die alle verschulden und auf Dauer spalten; wird sie als Komödie gelesen, kann das Lachen über die Leviten deren Gewalt entschärfen und vielleicht auch deren psychische und diskursive Wirkungen in ihr Gegenteil verkehren. 


\section{Literatur}

Albertz, Rainer. 2015. Exodus, Band II: Ex 19-40. Züricher Bibelkommentare AT 2.2. Zürich: TVZ.

Alighieri, Dante. 2001. La Commedia: Die Göttliche Komödie I. Inferno / Hölle. Stuttgart: Reclam.

Arendt, Hannah. 2002. Vita activa oder vom tätigen Leben. München: Pieper.

Assmann, Jan. 1998. Moses der Ägypter: Entzifferung einer Gedächtnisspur. München: C. Hanser.

Bachtin, Michail. 1969. Literatur und Karneval. Zur Romantheorie und Lachkultur. München: Hanser.

Badiou, Alain. 2010. „Die gegenwärtige Welt und das Begehren der Philosophie“. In Politik der Wahrheit, hg. v. Alain Badiou und Jacques Rancière, 7-36. Wien: Turia + Kant.

Bakunin, Michail. 1973. Selected Writings. London: Jonathan Cape Ltd.

Balibar, Étienne. 2006. Der Schauplatz der Anderen: Formen der Gewalt und Grenzen der Zivilität. Hamburg: Hamburger Edition.

Balke, Friedrich. 2009. Figuren der Souveränität. München: Fink.

Blanchot, Maurice. 2007. Die uneingestehbare Gemeinschaft. Berlin: Matthes \& Seitz.

Böckenförde, Ernst Wolfgang. 1985. „Politische Theorie und politische Theologie“. In Der Fürst dieser Welt: Carl Schmitt und die Folgen, hg. v. Jacob Taubes, 16-25. München: Fink.

Canetti, Elias. 1978. Masse und Macht. Düsseldorf: Claassen.

Deleuze, Gilles, und Félix Guattari. 1977. Anti-Ödipus: Kapitalismus und Schizophrenie I. Frankfurt am Main: Suhrkamp.

Deleuze, Gilles, und Félix Guattari. 1992. Tausend Plateaus: Kapitalismus und Schizophrenie II. Berlin: Merve.

Derrida, Jacques. 1972. Die Schrift und die Differenz. Frankfurt am Main: Suhrkamp.

Dostojewski, Fjodor Michailowitsch. 2016. Die Brüder Karamasow. Frankfurt am Main: Fischer.

Ernst, Christoph. 2020. „Hail, Trump! - Analogiedenken und Provokation als medienästhetische Formen im politischen Imaginären der US-amerikanischen Alt-Right-Bewegung“. In Trump und das Fernsehen: Medien, Realität, Affekt, Politik, hg. v. Dominik Maeder, Herbert Schwaab, Stephan Trinkaus, Anne Ulrich, Tanja Weber, 236-265, Köln: Herbert von Halem Verlag.

Feierstein, Liliana Ruth. 2011. „From the Other Side of the River: Borders in Jewish Culture and History“. In Topographien der Grenze: Verortungen einer kulturellen, politischen und ästhetischen Kategorie, hg. v. Christoph Kleinschmidt und Christine Hewel, 107-116. Würzburg: Königshausen und Neumann. 
Foucault, Michel. 1984. Von der Freundschaft als Lebensweise: Michel Foucault im Gespräch. Berlin: Merve.

Freud, Sigmund. 1921. Massenpsychologie und Ich-Analyse. Frankfurt am Main: Fischer.

Freud, Sigmund. 1939. Der Mann Moses und die monotheistische Religion: Drei Abhandlungen. Frankfurt am Main: Fischer.

Geiselberger, Heinrich, Hg. 2017. Die große Regression: Eine internationale Debatte über die geistige Situation der Zeit. Berlin: Suhrkamp.

Hénaff, Marcel. 2015. „Rätsel der Grausamkeit“. In Lettre International 109, Sommer 2015, 12-22. Berlin: Lettre International VerlagsGmbH.

La Boétie, Étienne de. 1924. Von der freiwilligen Knechtschaft. Berlin: Malik.

Lefort, Claude. 1990. „Die Frage der Demokratie“. In Autonome Gesellschaft und libertäre Demokratie, hg. v. Ulrich Rödel, 281-297. Frankfurt am Main: Suhrkamp.

Lefort, Claude. 1999. Fortdauer des Theologisch-Politischen? Wien: Passagen.

Lütjen, Torben. 2016. Die Politik der Echokammer: Wisconsin und die ideologische Polarisierung der USA. Bielefeld: Transcript.

Machiavelli, Niccolò. 1977. Discorsi. Gedanken über Politik und Staatsführung. Stuttgart: Kröner.

Maier, Heinrich. 2006. Was ist Politische Theologie?. München: Siemens Stiftung Sonderdruck.

Manemann, Jürgen. 2014. „Exoduspolitik - Politik ex memoria passionis“. In Das Politische und das Vorpolitische, hg. v. Michael Kühnlein, 339-36o. Baden-Baden: Nomos.

Marchart, Oliver. 2010. Die politische Differenz: Zum Denken des Politischen bei Nancy, Lefort, Badiou, Laclau und Agamben. Berlin: Suhrkamp.

Metz, Johann Baptist. 2016. „Politische Theologie“ in der Diskussion“. In Politische Theologie und Politische Philosophie, hg. v. Marie-Christine Kajewski und Jürgen Manemann, 67-101. Baden-Baden: Nomos.

Mouffe, Chantal. 2014. Agonistik: Die Welt politisch denken. Frankfurt am Main: Suhrkamp.

Nachtwey, Oliver. 2017. „Entzivilisierung. Über regressive Tendenzen in westlichen Gesellschaften“. In Die große Regression: Eine internationale Debatte über die geistige Situation der Zeit, hg. v. Heinrich Geiselberger, 215-231. Berlin: Suhrkamp.

Nadler, Steven. 1999. Spinoza: A Life. Cambridge: Cambridge University Press.

Nietzsche, Friedrich. 1999. „Zur Genealogie der Moral“. In Friedrich Nietzsche. Kritische Studienausgabe, hg. v. Giorgio Colli und Mazzino Montinari, 5. Band, München: dtv. 
Papadopoulos, Dimitris, Niamh Stephenson und Vassilis Tsianos. 2008. Escape Routes: Control and Subversion in the 21. Century. London: Pluto Press.

Plaut, W. Gunther, Hg. 200o. Die Tora in jüdischer Auslegung / 2, Schemot, Exodus. Gütersloh: Chr. Kaiser/Gütersloher Verlagshaus.

Plessner, Helmuth. 2003. „Grenzen der Gemeinschaft. Eine Kritik des sozialen Radikalismus“. In Helmuth Plessner. Gesammelte Schriften V. Macht und menschliche Natur, hg. v. Günter Dux, Odo Marquard und Elisabeth Ströker, 7-133, Frankfurt am Main: Suhrkamp.

Schmidt, Josef. 2013. Jakob - Echnaton - Moses: Propheten und Interpreten des einzigen Gottes. Hildesheim u. a.: Georg Olms Verlag.

Schmitt, Carl. 1925. Römischer Katholizismus und politische Form. München: Theatiner.

Schmitt, Carl. 1934. Politische Theologie: Vier Kapitel zur Lehre von der Souveränität. München und Leipzig: Dunker \& Humblot.

Schönberg, Arnold. 1954. Moses und Aron. Klavierauszug. Mainz: B. Schott’s Söhne.

Schweidler, Walter. 2011. „Bemerkungen zu den Begriffen ,politische Philosophie und ,politische Theologie“. In Politische Philosophie versus Politische Theologie? Die Frage der Gewalt im Spannungsfeld von Politik und Religion, hg. v. Wolfgang Palaver, Andreas Oberprantacher, Dietmar Regensburger, 43-51. Innsbruck: Innsbruck University Press.

Seebass, Horst. 1962. Mose und Aaron, Sinai und Gottesberg. Bonn: H. Bouvier und Co. Verlag.

Serres, Michel. 1987. Der Parasit. Frankfurt am Main: Suhrkamp.

Serres, Michel. 2008. Aufklärungen: Fünf Gespräche mit Bruno Latour. Berlin: Merve.

Spinoza, Baruch. 1984. Theologisch-politischer Traktat. Leipzig: Meiner.

Wacker, Bernd, und Jürgen Manemann. 2016. „Politische Theologie‘: Eine Skizze zur Geschichte und aktuellen Diskussion des Begriffs“. In Politische Theologie und Politische Philosophie, hg. v. Marie-Christine Kajewski und Jürgen Manemann, 9-54. Baden-Baden: Nomos.

Walzer, Michael. 1995. Exodus und Revolution. Frankfurt am Main: Fischer.

Wiedenhofer, Siegfried. 1976. Politische Theologie. Stuttgart: Kohlhammer. 